\title{
e-Migrinter
}

$21 \mid 2020$

Famille et migrations

\section{Pause pédagogique en Irak et en Syrie}

\section{Cyril Roussel et Tehem}

\section{(2) OpenEdition}

Journals

Édition électronique

URL : https://journals.openedition.org/e-migrinter/2526

DOI : 10.4000/e-migrinter.2526

ISSN : 1961-9685

\section{Éditeur}

UMR 7301 - Migrinter

\section{Référence électronique}

Cyril Roussel et Tehem, « Pause pédagogique en Irak et en Syrie », e-Migrinter [En ligne], 21 | 2020, mis en ligne le 26 mars 2021, consulté le 20 mai 2021. URL : http://journals.openedition.org/e-migrinter/ 2526 ; DOI : https://doi.org/10.4000/e-migrinter.2526 


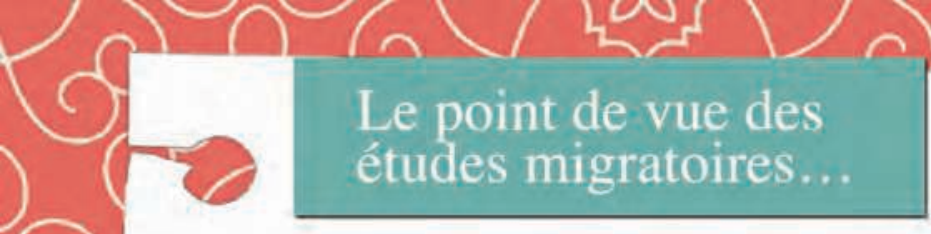

Il faut bien comprendre que le Moyen-Orient est une zone de tensions mondiales. Espace convoité, riche en ressources naturelles, mais aussi riche par sa diversité culturelle, cette région a été tellement violentée (guerres civiles, guerres par procuration, embargos, interventions étrangères, guerres contre le terrorisme...) qu'elle fournit aujourd'hui plus de la moitié des réfugiés de la planète. En Irak et Syrie, en plus des réfugiés, près de 9 millions de personnes se sont déplacées à l'intérieur de ces 2 pays depuis 2012.

Actuellement au Kurdistan d'Irak, on rencontre une multitude de personnes qui ont quitté leur région d'origine. Les déplacés sont originaires du reste de l'Irak et vivent pour partie dans des camps de toile ou dans des appartements loués. Beaucoup sont en « attente » dans la région autonome kurde. En septembre 2020, 261000 déplacés vivaient dans 43 camps à travers l'Irak sur près de 1,35 million de personnes déplacées. A la même période, environ 100000 réfugiés syriens, sur un total de 243000 , résidaient dans 10 camps, tous localisés dans la région autonome kurde.

La plupart des pays du Moyen-Orient n'ont jamais signé la convention de Genève sur les réfugiés et le droit d'asile. Ils ne sont donc pas contraints de proposer un statut juridique (dans le cadre du droit international) aux ressortissants des pays voisins qui fuient un conflit. Cette position s'explique par le contexte moyen-oriental marqué par des conflits à répétition. Cette région du monde a été grandement marquée par des vagues de déplacements massifs (génocide arménien, guerres d'Israël en Palestine, guerres du Liban, d'Irak et de Syrie) qui sont susceptibles de modifier localement les rapports de force démographique, économique et politique. Accueillir et offrir la nationalité ainsi qu'un travail à de telles masses de population n'est pas envisageable pour de si petits territoires. De plus, chercher à intégrer ces populations serait implicitement renier leur droit au retour et donc reconnaître des épisodes de « nettoyage ethnique ». Ainsi, les pays d'accueil préfèrent généralement considérer ces gens comme des " invités » en attente plutôt que comme des réfugiés qui pourraient rester à demeure.

Pour les réfugiés syriens par exemple, cette attente se prolongera tant que leur pays ne sera pas pacifié. S'ils ne peuvent prétendre à une protection juridique internationale, cela ne les empêche pas de s'inscrire auprès du HCR qui effectue un décompte des réfugiés. Le HCR est présent pour organiser l'aide humanitaire et coordonner l'action des ONG locales et internationales sur place. Et c'est dans les camps que l'aide humanitaire se concentre. Outre la possibilité d'y être hébergé sans frais, un réfugié pourra y avoir un accès aux soins et bénéficier d'un enseignement pour ses enfants. Avec des conflits régionaux qui durent, il est assez commun d'observer des allers-retours entre camp et ville (ou campagne) en fonction des possibilités financières des familles et donc de leur insertion sur le marché du travail local.

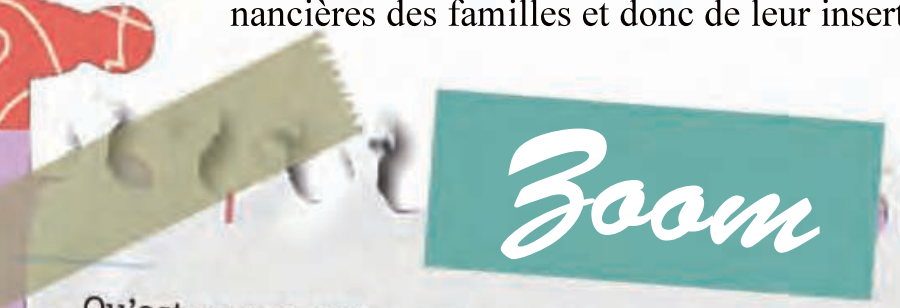

Qu'est-ce qui différencie un réfugié d'un travailleur mi tainement pas pertinente. le réfugié est celui qui vit dans un l'imaginaire occidental, qui a fui la guerre car il a tout perdu. faits, beaucoup ont fui avec leurs perdu. Mais dans les sion de potentielles violences ou bien parce que la situacherchent donc, tout commeinement dégradée. Ils le feraient, un travail dans le pays d'rants économiques vivre leur famille. Dans les faits, il d'accueil pour faire possible de faire la distinction faits, il est en général imchercher du travail et ceux quintre ceux qui partent contrainte (quelle contrainte? partent sous la chotomie " réfugié " / " migrant éconel ordre ?). La diopérante et fausse le débat car elle cró de personnes figées et les flux sont par deux catégories

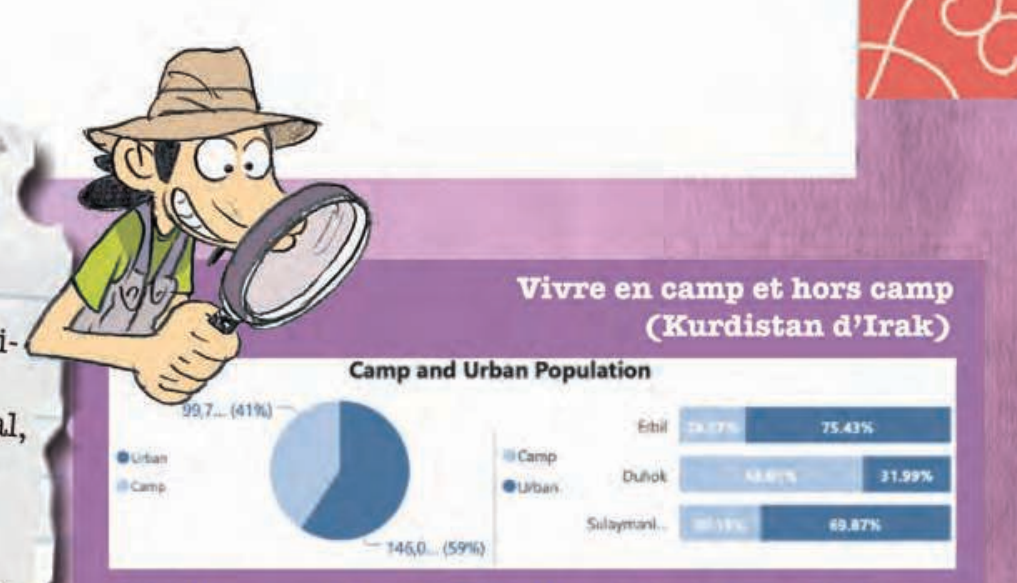

Sur l'ensemble du Kurdistan irakien, $59 \%$ des Syriens vivent en ville hors camp et $41 \%$ dans des camps. Erbil, où des milliers de Syriens sont employés, compte près de 125000 Syriens alors que seulement 30000 Syriens vivent dans les 4 camps en périphérie rurale. A Sulaymaniyah, la seconde ville kurde par son importance, les rapports sont de $70 \%$ en ville et $30 \%$ dans un camp. 
L'épuisement des ressources financières tant personnelles qu'humanitaires expliquent pourquoi des Syriens tentent de fuir les pays de premier asile (comme l'Irak) pour rejoindre l'Europe.

Les opportunités de quitter le Moyen-Orient de manière légale sont rares et ne concernent que quelques milliers de personnes par an sur les 5,5 millions de réfugiés syriens que compte le Moyen-Orient. Les passeurs acheminent, par des chemins illégaux, les candidats à l'exil vers la Turquie pour quelques centaines de dollars. De Turquie, des Syriens tentent, via des réseaux mafieux, de rejoindre l'Europe par des routes clandestines, parfois au prix de leur vie. Les plus aisés s'offre un voyage " sécurisé » et « garanti » comme le proposent certaines agences de passeurs. Dans ce business humain, le prix à payer pour éviter la traversée maritime est généralement d'une dizaine de milliers de dollars pour un périple en Europe dit " sécurisé », qui comprend de faux documents de voyage et un itinéraire en avion.

Passage de la frontière Irako-Syrienne Groupe de kurdes syare la région autonome Syrie pour rejl Ils attendent qu'une embarcation vienne les chercher pour traverser le Tigre qui marque à cet endroit la frontière entre les deux pays. Dréféré se déplamilliers de familles fuir un pays qui voyait cer en Irak pour son économie s'écrouler. jour après jour son el Cyril, février 2014.

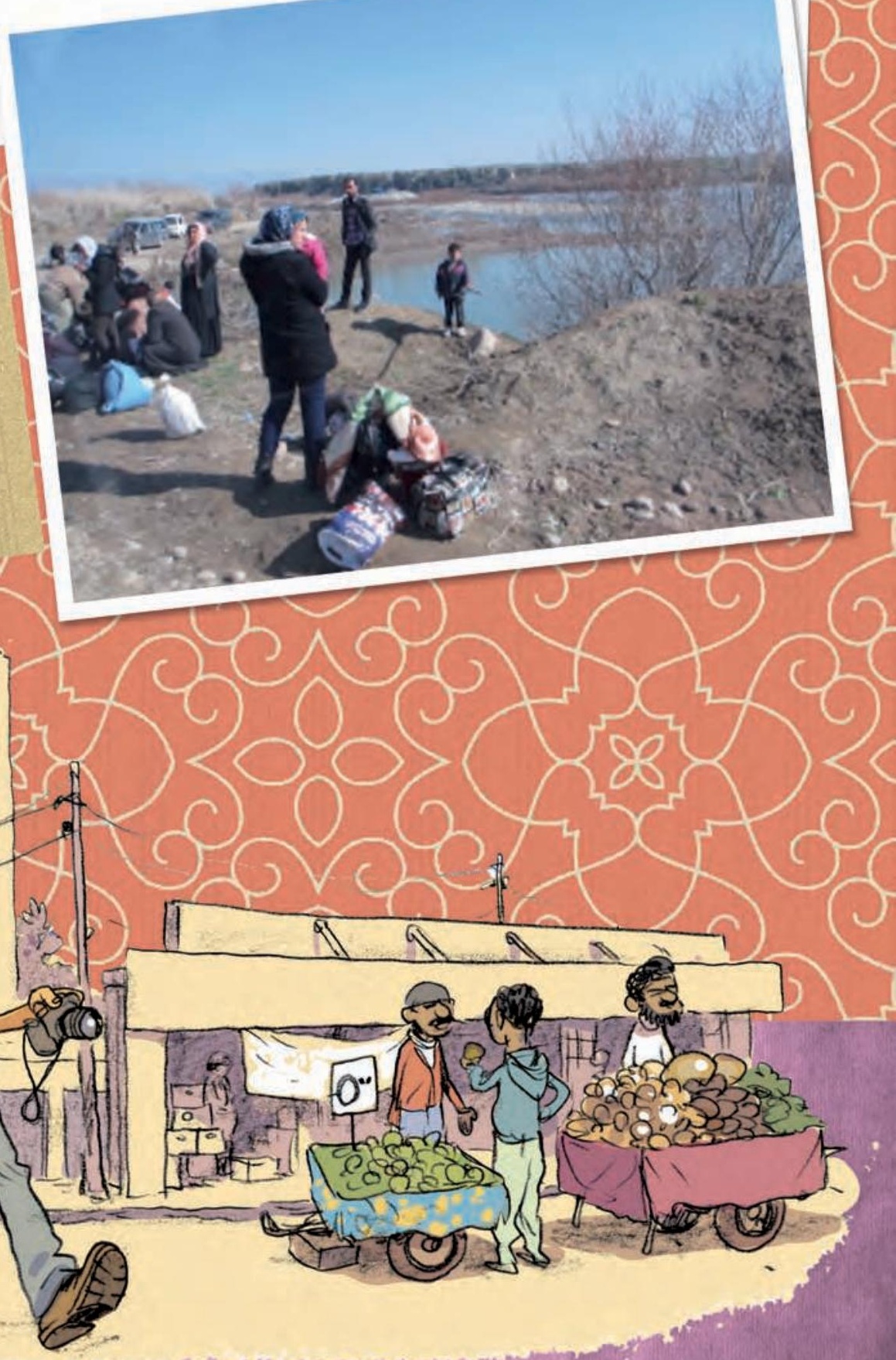




\section{$15,-6$ \\ Voyage, voyage}

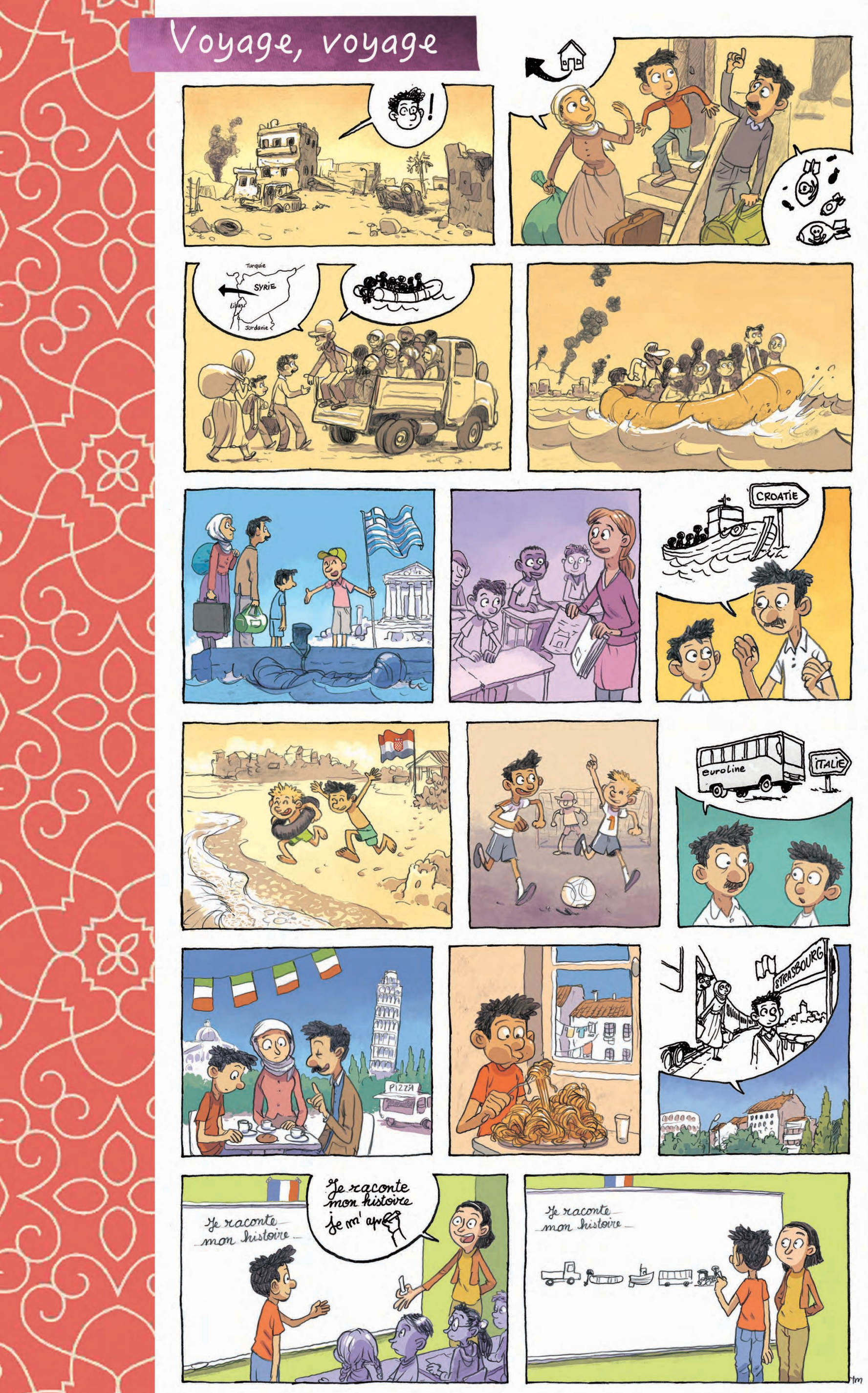

R.
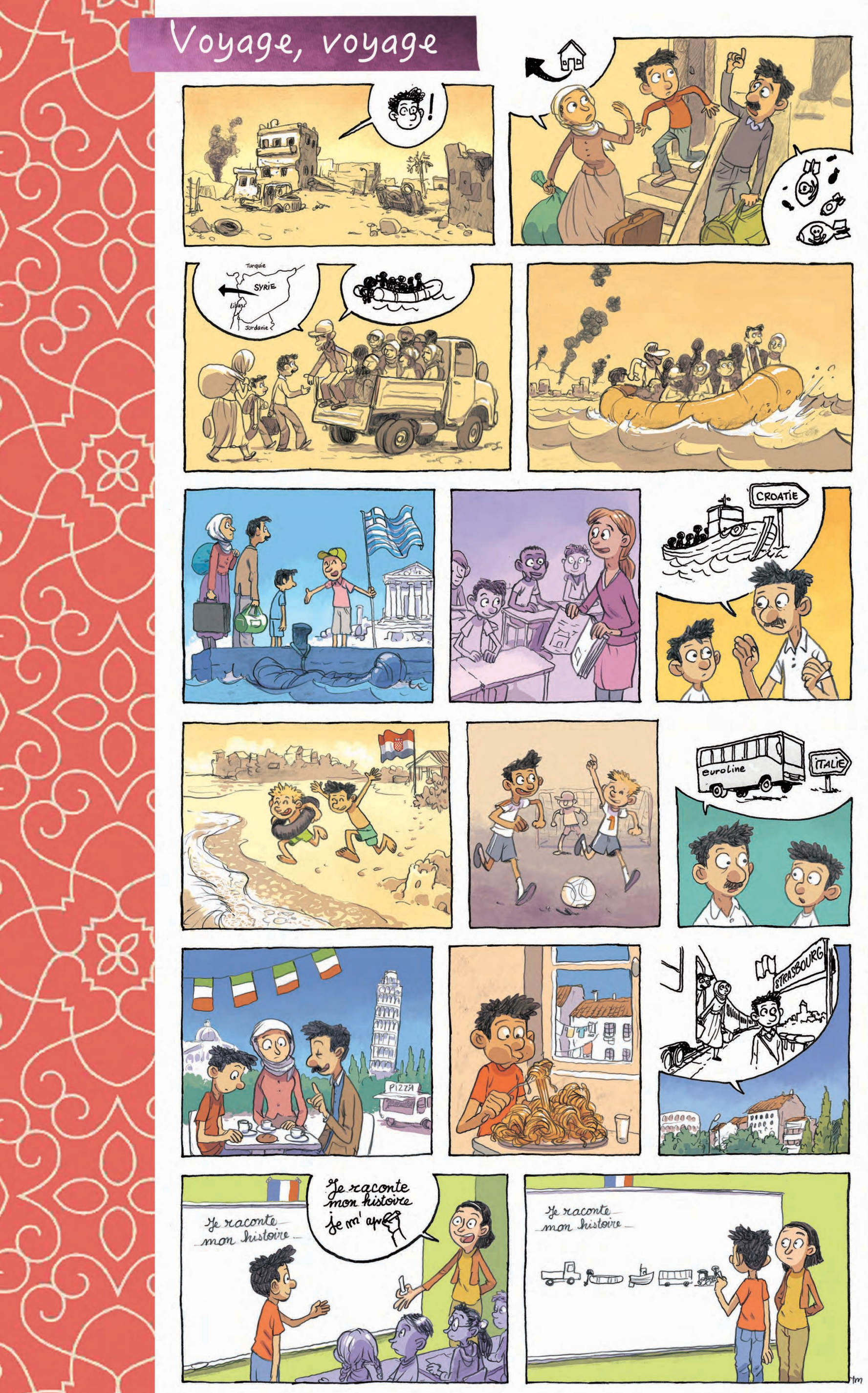
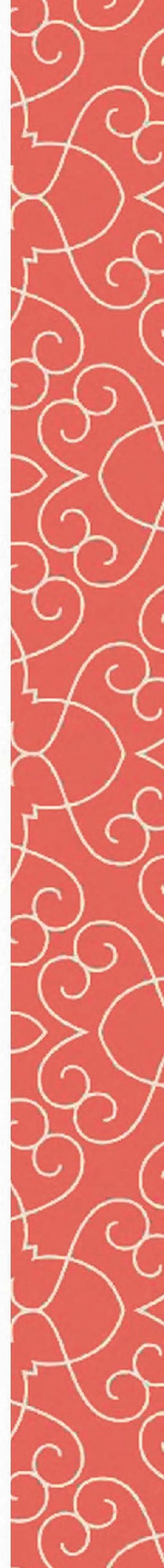
Réfugié : Ce terme est utilisé de manière généraliste pour désigner des personnes qui se trouvent dans des situations bien différentes. Dans le cadre juridique du droit international, un réfugié est celui qui a obtenu le droit d'asile, donc une protection qui lui procure un statut. Mais dans de nombreux pays, le droit international ne s'applique pas. Ainsi de nombreux Etats, comme la plupart des pays du Moyen-Orient, appliquent une protection qui leur est propre. Via un système de sponsoring (nommé kafala dans les pays arabes), les réfugiés peuvent sortir des camps, travailler et se loger dans les centres urbains. En général, les autorités locales ferment les yeux sur le travail clandestin de ces personnes, ce qui arrange tout le monde.

Attente: Dans un processus de migration forcée, l'attente des personnes exilées dans les pays de premier asile est directement reliée à la durée et à l'évolution du conflit dans le pays de départ. Tant que les conditions du retour ne sont pas assurées (combats, contrôle par des milices hostiles, destruction de l'habitat, occupation des terres et des logements par des populations déplacées à l'intérieur du pays en guerre, etc.), les réfugiés ne peuvent rentrer. Dans le cas syrien, l'attente en Irak se prolonge depuis près de 10 ans. Cela implique de développer des stratégies de survie dans leurs lieux d'accueil.

Migration illégale: Il s'agit d'une migration qui cherche à éviter les points de contrôle étatique (postes douaniers aux frontières terrestres et aériennes) pour quitter un pays et se rendre dans un autre. Elle s'effectue grâce à l'aide de passeurs qui connaissent bien les points de contrôle et qui peuvent soit les contourner, soit négocier avec les personnels de sécurité. Elle peut se réaliser aussi à l'aide de faux documents de voyage afin de tromper la vigilance des autorités qui contrôlent l'accès aux territoires. Par terre, par mer et dans les airs, elle alimente une économie souterraine très prospère où tout se paye au prix fort.

\section{J'approfondis...}

Doraï, Kamel ; Roussel, Cyril (2015) Crises des États au Moyen-Orient : Conflits, circulations et migrations forcées, In Schmoll, C. ; Thiollet, H. ; Wihtol de Wenden, C. (dir.) Migrations en Méditerranée : Permanences et mutations à l'heure des révolutions et des crises, Paris, CNRS Éditions, pp. 113-124. Roussel, Cyril (2009) Migrations et réfugiés au Proche-Orient et au Soudan - Introduction, Revue Géographique de l'Est, Vol. 49, nº [Disponible sur Internet] Consulté le 23 octobre 2018. URL: https://journals.openedition.org/rge/1968.

Comme on le voit, derrière ce mot valise de réfugié, se cache des personnes et des familles qui doivent tout simplement, reconstruire leur vie dans un pays qui n'est pas le leur. II sy cache aussi des histoires de vie très diverses qui ne sont pas forcément toujours dramatiques, comme on pourraits $y$ attendre. En, fait, en parlant de « migrations forcées》, on imagine, car c est l image largement véhiculée, des personnes paurres et démunies qui fuient des massacres. Si c est parfois malheureusement le cas, il ne faut pas fermer les yeux sur. I'immense diversité des cas. Le réfugié est avant tout un migrant et donc un acteur autonome qui décide du moment de son grant et donc un act son parcours en exil en fonction des contraintes et des ressources dont il dispose (ressources financières, réseaux de connaissances, opportunités de travail...). Nous retiendrons enfin que même si les conflits sortent du, spectre médiatique, des millions de personnes vivent dans l' attente d'un retour imprévisible; la durée indéfinie de l'exil rend difficile toute perspective $d^{\prime}$ avenir, favorisant, dans le même temps, le recours aux routes migratoires clandestines, seule option de fuite.

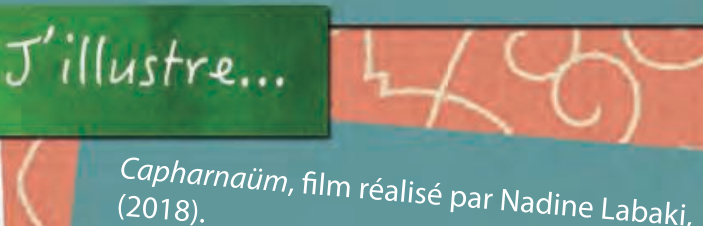

Les deux réfugiés, Pièce de théâtre des frères Malas (2017). https://www.youtube.com/watch? vixsq7oTRYbA Lodyssée d'Hakim, BD en trois tomes réalisée Dabien Toulmé (2018, 2019 et 2020).

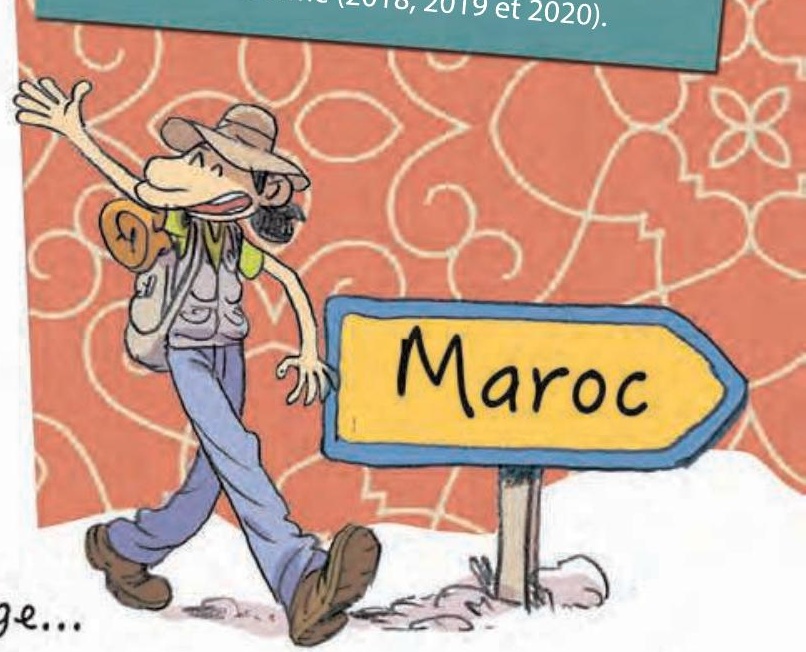

